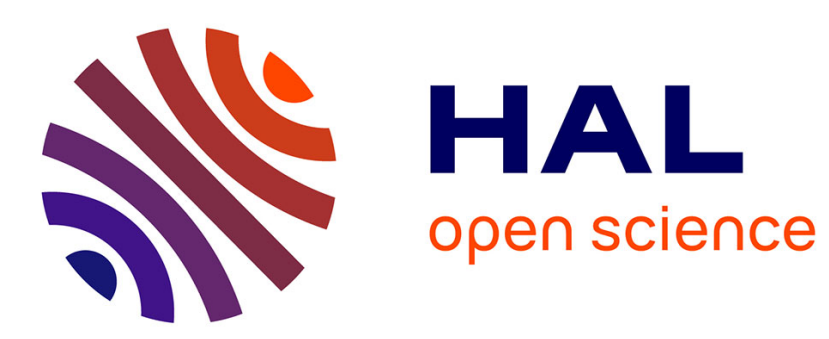

\title{
INTERPRÉTATION DES MESURES MAGNÉTIQUES EFFECTUÉES SUR HoFeO3
}

\author{
Y. Allain, J. Bouree, J. Denis, J. Wajnflasz, M. Lecomte
}

\section{To cite this version:}

Y. Allain, J. Bouree, J. Denis, J. Wajnflasz, M. Lecomte. INTERPRÉTATION DES MESURES MAGNÉTIQUES EFFECTUÉES SUR HoFeO3. Journal de Physique Colloques, 1971, 32 (C1), pp.C1-494-C1-495. 10.1051/jphyscol:19711163 . jpa-00213984

\section{HAL Id: jpa-00213984 https://hal.science/jpa-00213984}

Submitted on 1 Jan 1971

HAL is a multi-disciplinary open access archive for the deposit and dissemination of scientific research documents, whether they are published or not. The documents may come from teaching and research institutions in France or abroad, or from public or private research centers.
L'archive ouverte pluridisciplinaire $\mathbf{H A L}$, est destinée au dépôt et à la diffusion de documents scientifiques de niveau recherche, publiés ou non, émanant des établissements d'enseignement et de recherche français ou étrangers, des laboratoires publics ou privés. 


\title{
INTERPRÉTATION DES MESURES MAGNÉTIQUES EFFECTUÉES SUR $\mathrm{HoFeO}_{3}$
}

\author{
Y. ALLAIN, J. E. BOUREE, J. DENIS, J. WAJNFLASZ, M. LECOMTE \\ Service de Physique du Solide et de Résonance Magnétique \\ Centre d'Etudes Nucléaires de Saclay BP no 2, 91 Gif-sur-Yvette
}

Résumé. - Un modèle d'Ising associé aux deux directions de très forte anisotropie du moment de $\mathrm{H}_{0}^{3+}$ dans $^{3} \mathrm{HoFeO}_{3}$ permet de rendre compte des résultats expérimentaux d'anisotropie et d'aimantation. Une théorie phénoménologique qui prévoit une zone de température de réorientation des moments $\mathrm{Fe}^{3+}$ semble être confirmée par les résultats d'aimantation suivant [001] et les mesures de chaleur spécifique.

Abstract. - An Ising model related to the hard directions of the magnetic moment of $\mathrm{Ho}^{3+}$ in $\mathrm{HoFeO}_{3}$ explains our anisotropy and magnetization data. The specific heat and the magnetization measurements along [001] confirm a phenomenological theory which predicts a reorientation temperature range for the $\mathrm{Fe}^{3+}$ moments.

Dans le cadre de l'étude de la réorientation des moments de l'orthoferrite d'holmium $\mathrm{HoFeO}_{3}$ vers $50^{\circ} \mathrm{K}$, nous avons entrepris une étude détaillée des propriétés magnétiques à diverses températures sur un échantillon monocristallin. En résumé [1] [2], il apparaît à $643^{\circ} \mathrm{K}$ un ordre antiferromagnétique non colinéaire des moments du fer dans le plan (010), le moment résultant étant dans la direction [001]; vers $50 \mathrm{~K}$, ce moment se réoriente dans la direction [100] en induisant un champ effectif $H_{\mathrm{Fe}}$ qui polarise les ions $\mathrm{Ho}^{3+}$ dans deux directions de très forte anisotropie ; à très basse température il apparaît probablement un ordre des holmium. Nos résultats expérimentaux ont apporté des précisions nouvelles.

1. SUSCEPTIBILITÉ MAGNÉTiQUE. - Les courbes de susceptibilité initiale représentées figure 1 sont relatives au sous-réseau $\mathrm{Ho}^{+3}$; la constante de Curie pour $T>100{ }^{\circ} \mathrm{K}$ est la même dans les trois directions et correspond à l'ion libre $\mathrm{Ho}^{3+}$. La susceptibilité dans

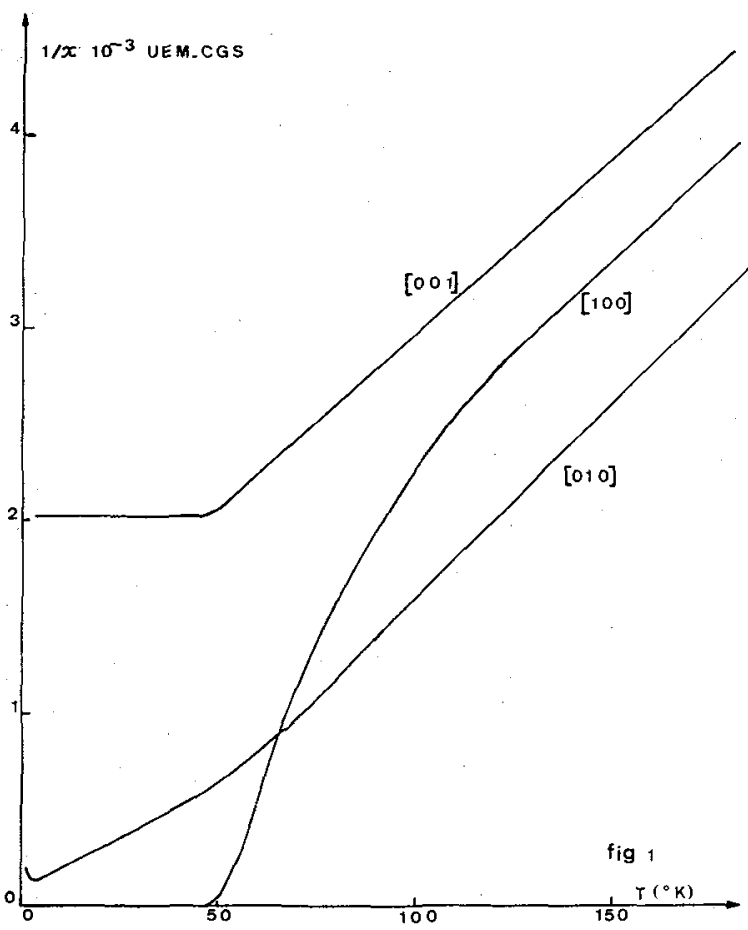

Fig. 1. - Susceptibilité initiale du sous-réseau $\mathrm{Ho}^{3+}$. la direction [001] confirme la configuration (Fig. 2) pour $T<50^{\circ} \mathrm{K}$ des moments holmium dans le plan de très forte anisotropie (001). Dans la direction [100] il apparaît un moment permanent vers $50^{\circ} \mathrm{K}$. L'hypothèse de deux directions de forte anisotropie explique la loi de variation de la susceptibilité initiale suivant [010]: à 0 \% elle serait nulle; expérimentalement elle commence à diminuer au-dessous de $3 \mathrm{oK}$ environ.
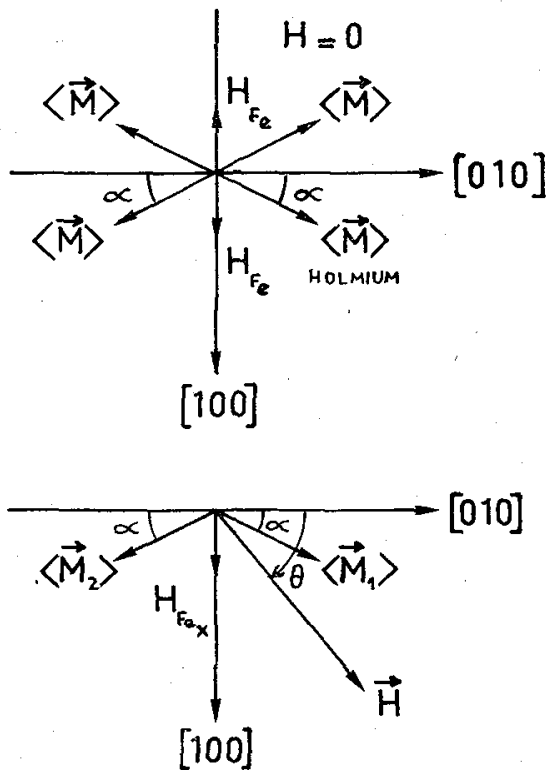

FIG. 2. - Configuration des moments dans le plan (001) pour $T<50^{\circ} \mathrm{K}$.

2. Amantation. - La loi d'aimantation suivant [100] est celle de l'ion $\mathrm{Ho}^{3+}$ soumis au champ extérieur auquel vient s'ajouter le champ effectif $H_{\mathrm{Fe}}$ provenant du sous-réseau fer, les moments des holmium étant maintenus très fortement dans la direction $\Delta$ (un champ de $200 \mathrm{kG}$ les écarte très peu de cet axe). En champ nul nous avons deux domaines où les moments résultants des $\mathrm{Ho}^{3+}$ sont opposés (Fig. 2) ; l'énergie de paroi est faible : une dizaine de gauss appliqués dans la direction [100] suffit pour faire coincider les moments. Les résultats obtenus suivant [010] (Fig. 3) sont conformes à la configuration des moments, sauf en ce qui concerne l'anomalie qui apparaît vers 


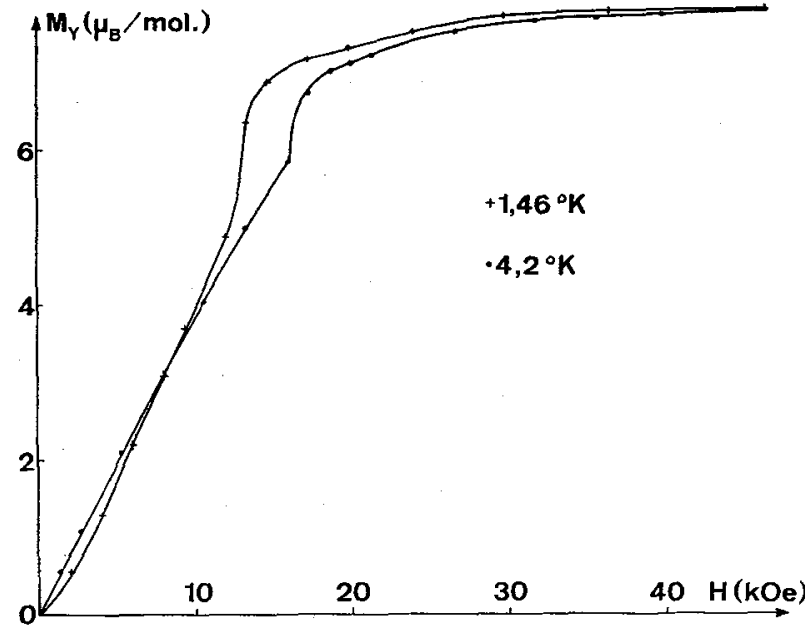

FIG. 3. - Aimantation dans la direction [010].

$12 \mathrm{kOe}$ et qui consiste en une brusque variation de l'aimantation ; il est possible qu'elle soit due à un phénomène de magnétostriction accompagné d'une variation du champ effectif avec les distances interatomiques.

3. ANISOTROPIE (Fig. 4). - L'anisotropie du cristal nous a amenés à faire des mesures du couple en fonc-

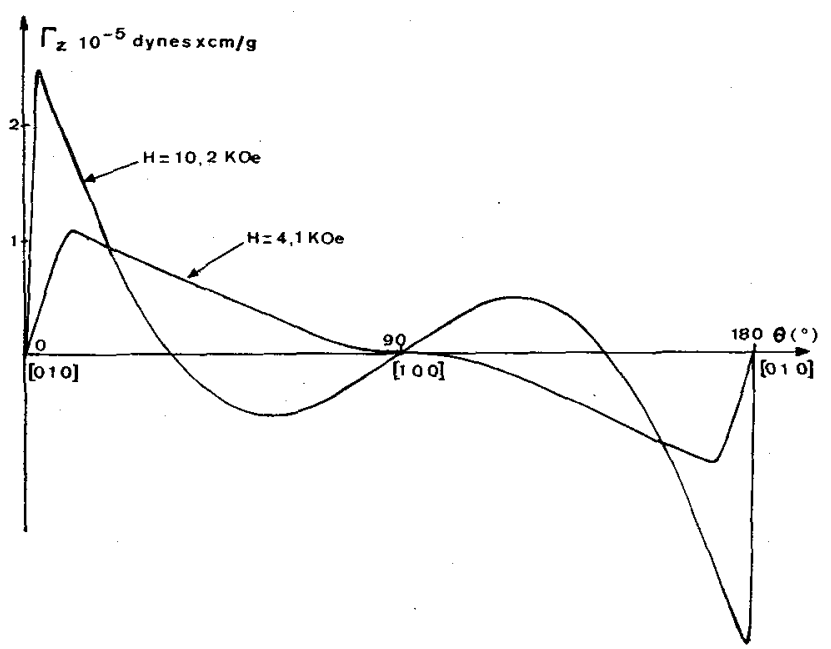

FIG. 4. - Exemples de couples d'anisotropie $\left(T=15^{\circ} \mathrm{K}\right)$.

tion de $T$ et $H$. Seul le plan (001) a été jusqu'à présent étudié.

Interprétation. - Dans ce but nous avons fait deux hypothèses fondamentales:

1) Les directions $\Delta$ ne changent pas avec la température; ceci semble être confirmé par la diffraction des neutrons [3] [4].

2) L'existence de deux directions de forte anisotropie nous a suggéré un modèle d'Ising pour la configuration holmium.
Si $H$ fait un angle $\theta$ avec [010] les moments des holmium s'écrivent alors :

$$
\begin{aligned}
<M_{1}>= & g \mu_{\mathrm{B}} S \text { th }\left\{\frac{g \mu_{\mathrm{B}} S}{k T}[H \cos (\theta-\alpha)+\right. \\
& \left.\left.+H_{\mathrm{Fe}} x \sin \alpha\right]-\frac{8 S J_{2} \cos ^{2} \alpha}{g \mu_{\mathrm{B}} k T}<M_{2}>\right\} \\
<M_{2}>= & g \mu_{\mathrm{B}_{-}} S \text { th }\left\{\frac{g \mu_{\mathrm{B}} S}{k T}[-H \cos (\alpha+\theta)+\right. \\
& \left.\left.+H_{\mathrm{Fe}} x \sin \alpha\right]-\frac{8 S J_{2} \cos ^{2} \alpha}{g \mu_{\mathrm{B}} k T}<M_{1}>\right\}
\end{aligned}
$$

et le couple :

$$
\begin{aligned}
& \Gamma_{\text {[001] }}= \\
& =H\left[\left\langle M_{1}\right\rangle \sin (\alpha-\theta)+\left\langle M_{2}\right\rangle \sin (\alpha+\theta)\right] .
\end{aligned}
$$

Les calculs faits à la machine à l'aide d'un programme de régression optimale non linéaire [5] ont montré la compatibilité entre toutes les valeurs expérimentales (l'anomalie suivant [010] mise à part) et ont donné les valeurs suivantes pour les paramètres ajustables:

$$
\begin{gathered}
H_{\mathrm{Fe}} x(T)=19,5 \mathrm{kOe}\left( \pm 1 \text { Oe) à } 20^{\circ} \mathrm{K}\right. \\
\alpha=24^{\circ}\left( \pm 30^{\prime}\right) ; g=16,8 \\
J_{2} / k=-0,8^{\circ}( \pm 0,3)
\end{gathered}
$$

Une théorie phénoménologique où on introduit dans l'énergie libre les termes d'anisotropie du premier et du second ordre et un terme d'interaction holmiumfer [6] [7] avec l'hypothèse d'une forte anisotropie de $g$ dans le plan (001) explique la réorientation des spins entre deux températures $T_{1}$ et $T_{2}$. La décroissance sensible de l'aimantation suivant [001] au-dessous de $65{ }^{\circ} \mathrm{K}$ et les résultats de chaleur spécifique (Fig. 5)

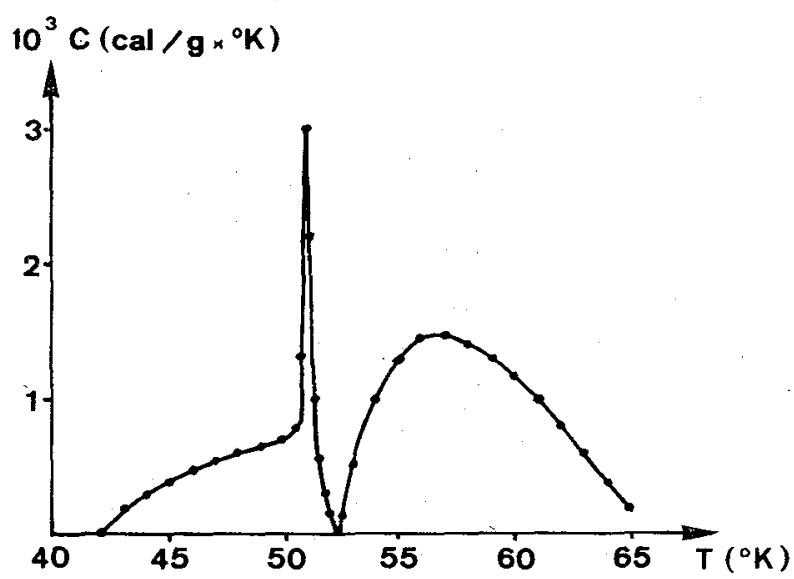

Fig. 5. - Chaleur spécifique relative au phénomène de réorientation $\left(\Delta S \simeq 0,5\right.$ Joule/mole $\left.\times{ }^{\circ} \mathrm{K}\right)$.

semblent confirmer l'existence d'une zone de température de réorientation [8] $\left(50^{\circ} \mathrm{K}-65^{\circ} \mathrm{K}\right)$. Signalons que la forte anisotropie de $g$ a été récemment interprétée [9] en considérant deux singulets voisins suffisamment éloignés des autres états.

\section{Bibliographie}

[1] Bozorth (R. M.) et al., Phys. Rev. Letters, 1958, 1, 3. [2] Allain (Y.) et al., C. R. Ac. Sci., Paris, 1969, 269, 1024.

[3] Koehler (W. C.) et al., Phys. Rev., 1960, 118, 58.

[4] Meriel (P.), Communication privée, CEN-Saclay.

[5] Tournaire (M.), J. Physique, 1969, 30, 737.
[6] Bertaut (E. F.), Magnetism III, Rado and Suhl, 179.

[7] Sivardière (J.), Solid State Comm.; 1969, 7, 1555.

[8] Bourée (J. E.), Thèse 3 e cycle, Paris, 1970.

[9] Malozemoff (A. P.) and White (R. L.), Solid State Comm., 1970, 8, 665 . 